\title{
《中国药典》动物药材基原物种中文名和 拉丁学名引证规范
}

\author{
蒋超", 黄璐琦, 袁媛", 李军德
}

中国中医科学院中药资源中心, 道地药材国家重点实验室培育基地, 北京 100700

*联系人, E-mail: jiangchao0411@126.com; y_yuan0732@163.com

收稿日期：2017-11-16; 接受日期：2018-04-11; 网络版发表日期：2018-06-20

中央本级重大增减支项目(批准号: 2060302)、中央级公益性科研院所基本科研业务费专项资金(批准号: ZZ10-008)和中国中医科学院中药资 源中心自主选题(批准号: ZZXT201709)资助

\begin{abstract}
摘要 《中国药典》是我国药品监管的法定技术标准, 作为保障药品质量的法典, 具有科学性、规范性和权威性. 然而, 历版药典一直未对收录的动物药材基原物种的中文名和拉丁学名进行审查和修订，《中国药典》中一些遗 留的基原物种名称引证不规范现象不断积累。作者根据《中国动物志》、《动物学中适用名称目录》以及国际 权威动物名录数据库进行比较, 并核对原始文献, 统计分析了 2015年版《中国药典》一部和四部收录的动物药材 基原物种学名和中文名引证不规范的现象, 并提出修正建议, 旨在引起中药研究者的重视, 维护药典的权威性和 规范性.
\end{abstract}

关键词《中国药典》, 动物药材, 拉丁学名, 中文名

《中华人民共和国药典》(以下简称《中国药 典》)是我国药品监管的法定技术标准, 作为保障药品 质量的法典, 具有科学性、规范性和权威性. 动物药材 是中医药的重要组成部分，2015年版《中国药典》共 收载动物药材 102 味, 其中一部收载动物药材 52 味, 四 部“成方制剂中本版药典未收载的药材和饮片”项下收 载动物药材 50 味, 来源于 103 种基原动物并附有相应的 拉丁学名 ${ }^{[1,2]}$. 拉丁学名的使用确保了各基原物种的唯 一性, 为中药生产、流通、应用和检验提供了规范. 然 而，自1953年首版《中国药典》颁布以来动物药材品 种不断增加, 国家医药管理部门始终未对收录的动物 药材各基原物种的中文名和拉丁学名进行全面审查和
修订, 《中国药典》中一些遗留的基原物种名称引证 不规范现象不断积累, 已开始影响《中国药典》的 施行.

本文将2015年版《中国药典》一部和四部中的动 物药材基原物种拉丁学名与《中国动物志》、国际动 物命名法规委员会建立的《动物学中适用名称目录》 (Official Lists and Indexes of Names and Works in Zoology ${ }^{[3]}$, Species 2000的生物动态名录ITIS Catalogue of Life (COL, http://www.catalogueoflife.org/col/), Global Names Architecture (GNA, http://globalnames. org/), Encyclopedia of Life (http://www.eol.org/), Global Biodiversity Information Facility (GBIF, http://www.

引用格式: 蒋超, 黄璐琦, 袁媛, 等. 《中国药典》动物药材基原物种中文名和拉丁学名引证规范. 中国科学: 生命科学, 2018, 48: 772-782

Jiang C, Huang L Q, Yuan Y, et al. Standard citations of zoological scientific names and Chinese names in Chinese Pharmacopoeia (in Chinese). Sci Sin Vitae, 2018, 48: 772-782, doi: 10.1360/N052017-00254 
gbif.org/), FishBase (http://fishbase.org/search.php), World Register of Marine Species (WoRMS, www. marinespecies.org), Cockroach Species File Online (http://cockroach.speciesfile.org/), Amphibian Species of the World (http://research.amnh.org/vz/herpetology/ amphibia/)等国际权威动物名录数据库进行比较, 同时 追溯物种初始发表文献, 分析了《中国药典》中动物 药材基原物种学名引证不规范之处, 提出修正建议, 以及相应的中文名称修改建议 ${ }^{[4]}$.

\section{1 动物学名与国际动物命名法规}

动物学名是该动物在全世界统一的称谓, 受国际 动物命名法规(The International Code of Zoological Nomenclature, ICZN)的规范 ${ }^{[5,6]}$. 国际动物命名法规采 用双名法命名规则，规定最早的、合乎各项规则的拉 丁文名称, 即为该动物的学名(scientific name). 现行第 四版国际动物命名法规包含 18 章共 90 条，与学名引证 有关的主要包括双名法原则(principle of binominal nomenclature)、同等原则(principle of coordination)、优 先权原则(principle of priority)、第一修订人原则(principle of the first reviser)、异物同名关系原则(principle of homonymy)、第22条日期的引证、第51条命名者姓 名的引证及第79条《动物学中适用名称目录》.

其中, 第5 条“双名法原则”规定, 动物物种 (species) 学名由两个名称组成, 即属名+种加词, 亚种(subspecies)学名由 3 个名称组成, 为属名 + 种加词 + 亚种名. 习 惯上, 为了科学、规范、准确地指明一种动物, 通常在 种加词或亚种名后面加上命名人和发表年份.

第23条“优先权原则”规定, 一个分类单元的有效 名称是最早用于该分类单元的适用名称，除非因国际 动物命名法规的规定或委员会的裁定或另一名称地位 优先而失效. 第24条“第一修订人原则”规定, 各名称的 优先地位按其发表时间自动确定，当几个命名举动的 优先地位不能客观决定时, 由首位修订人确定.

第52条“异物同名关系原则”规定，当两个或多个 分类单元可彼此清楚分辨时，不能使用同一名称来指 示. 异物同名时使用优先权原则决定的较早名称为有 效名称，科、属、种或亚种名称即使仅一个字母的差 异也并非同名关系.

第79条《动物学中适用名称目录》规定，凡是目
录中已被采纳的“部分名录”中出现的一个名称，即视 为适用名称，其拼法、日期和命名者身份即为目录中 所记载者; 凡 “部分名录”中出现的一个名称所指的分 类单元，即被视为具名模式; 凡未列入目录的同一分 类单元的异名, 在动物命名法上无任何地位.

根据国际动物命名法规的基本原则和建议(第22 条 $\mathrm{A}$, 第 51 条 $\mathrm{A} \sim \mathrm{G})$, 为分辨异物同名, 规范而准确地指 明一种动物物种, 应正确引用所指明物种的学名(包括 其拼写), 在涉及分类单元著作物中, 需引证其名称命 名者和命名日期至少一次. 现行 2015 年版《中国药 典》动物药材基原物种拉丁学名存在较多与国际动物 命名法规不符或引证失实之处.

\section{2 《中国药典》存在的引证失误}

\section{1 拼写错误}

根据多个数据库的交叉核对，并查阅原始发表文 献, 确定《中国药典》103种基原动物学名引证中至 少存在20处拼写错误, 包括属名拼写错误, 例如, 鹿心 粉基原动物梅花鹿(Cevus nippon Temminck)的属名应 当是“Cervus”, 熊胆粉基原动物黑熊(Selenaretos thibetanus Cuvier)的属名应当是“Selenarctos”; 种加词拼写 错误, 例如, 石决明基原澳洲鲍(Haliotis ruber (Leach)) 的种加词应当是 “rubra”, 水蛭基原动物水蛭(Hirudo nipponica Whitman)种加词应当是“nipponia”; 命名人 拼写错误, 例如, 水牛角浓缩粉基原动物水牛(Bubalus bubalis Linnaens)的命名人林奈拼写应当是Linnaeus, 方海(螃蟹)基原动物中华线毛螯蟹(Eriocheir sinensis H. Miline-Edwalds)的命名人爱德华兹的拼写应当是 H. Milne Edwards, 详见表1.

\section{2 同名和异名}

根据国际动物命名法规“优先权原则”，基原动物 的正确学名为第一个符合各项规定的拉丁学名. 晚出 名包括异名和同名, 晚出异名根据法规应废弃; 晚出 同名除导致引证不实外, 还可能会产生异物同名, 致 使基原错误. 例如1916年, Matsumura命名了虹虫的基 原动物复带虻(Tabanus bivittatus Matsumura), 而1846 年Macquart同样命名了Tabanus bivittatus Macquart, 在 简写不引证命名人的情况下将导致基原错误. 除牛、 猪、家鸡、鹅等驯养动物外，2015年版《中国药典》 
表 1 《中国药典》中动物药材基原物种学名拼写错误a)

\begin{tabular}{|c|c|c|c|}
\hline NO. & 品名 & 《中国药典》学名 & 正确拼写 \\
\hline 1 & 鹿心粉 & 梅花鹿(Cevus nippon Temminck) & Cervus nippon Temminck \\
\hline 2 & 熊胆粉 & 黑熊(Selenaretos thibetanus Cuvier) & Selenarctos thibetanus (Cuvier) \\
\hline 3 & 海龙 & ᄀ海龙(Solenognathus hardwickii (Gray)) & Solegnathus hardwickii (Gray) \\
\hline 4 & 刺猬 & 短刺猬(Hemichianus dauricus Sundevol) & Hemiechinus dauricus (Sundevall) \\
\hline 5 & 猪胆粉 & 猪(Sus scrofadomestica Brisson) & Sus scrofa domestica Brisson \\
\hline 6 & 蜂房 & 果马蜂(Polistes olivaceous (DeGeer)) & Polistes olivaceus (DeGeer) \\
\hline 7 & 水蛭 & 水蛭(Hirudo nipponica Whitman) & Hirudo nipponia Whitman \\
\hline 8 & 哈蟆油 & 中国林蛙(Rana tem poraria chensinensis David) & Rana temporaria chensinensis David \\
\hline 9 & 蛇肉 & 高原蝮(Agkistrodon strauchii bedriaga) & Agkistrodon strauchi bedriaga \\
\hline 10 & 石决明 & 澳洲鲍(Haliotis ruber (Leach)) & Haliotis rubra Leach \\
\hline 11 & 虫白蜡 & 白蜡虫(Ericerus pela (Chavannes) Guerin) & Ericerus pela (Chavannes) Guérin \\
\hline 12 & 方海(螃蟹) & 云南溪蟹(Potamon (Potamon) yunanensis) & Potamon yunnanensis Kemp \\
\hline 13 & 方海(螃蟹) & 中华线毛螯蟹(Eriocheir sinensis H. Miline-Edwalds) & Eriocheir sinensis $\mathrm{H}$. Milne Edwards \\
\hline 14 & 猪蹄甲 & 猪(Sus scrofa domestiea Brisson) & Sus scrofa domestica Brisson \\
\hline 15 & 水牛角浓缩粉 & 水牛(Bubalus bubalis Linnaens) & Bubalus bubalis (Linnaeus) \\
\hline 16 & 石燕 & 中华弓石燕(Cyrtiospirifer sinensis (Graban)) & Cyrtospirifer sinensis (Grabau) \\
\hline 17 & 鸡蛋壳(炒) & 家鸡(Gallus gallus domesticus Brissum) & Gallus gallus domesticus Brisson \\
\hline 18 & 蛇蜕 & 王锦蛇(Elaphe carinata (Guenther)) & Elaphe carinata (Günther) \\
\hline 19 & 蛇肉 & 翠青蛇(Opheodrys major (Guenther)) & Opheodrys major (Günther) \\
\hline 20 & 我胆粉 & Anser cygnoides dommestica Brisson & Anser cygnoides domestica Brisson \\
\hline
\end{tabular}

a) 虽然命名人中注音符号花与 $\mathrm{u}$ 具有等同性, 但《中国药典》中引证有䩪蛇(Agkistrodon acutus (Güenther)和多欶海盘车(Asterias amurensis Lütken), 故将所有命名人中 $\mathrm{u}$ 统一为

还在海马、海螵蛸、蝉蜕、鹿香、夜明砂、虹虫等项 下存在至少5项晚出名(表2).

\section{3 组合改变导致的引证错误}

根据国际动物命名法规，动物学名经重新组合而 不在原来的属时，引证时需将种群的命名者和日期置 圆括号内. 2015年版《中国药典》中存在20项组合改 变但未加圆括号导致的引证错误(表3). 此外石决明基 原动物之一的澳洲鲍(Haliotis ruber (Leach))和白鲍 (Haliotis laevigata (Donovan))自命名以来一直置于鲍 属, 组合从未改变, 故其学名不应加圆括号而是 Haliotis rubra Leach和Haliotis laevigata Donovan.

\section{4 命名人引证失实}

（1）命名人引证错误. 《中国药典》中的马稘 (Moschus sifanicus Przewalski) 为动物学家Büchner在
Die Säugethiere der Ganssu-Expedition. (1884 1887)上 合格发表, 目前尚无证据表明普热瓦尔斯基(Przewalski)发表过新物种Moschus sifanicus.

(2) 命名人缺失. 共两项, 《中国药典》中方海(螃 蟹)的基原动物溪蟹Potamon (Potamon) denticulata和 云南溪蟹Potamon (Potamon) yunanensis缺失命名人的 引证, 且引证了亚属名, 其完整学名应当为Potamon (Potamon) denticulata H. Milne Edwards, 1853和 Potamon (Potamiscus) yunnanensis Kemp, 1923.

(3) 命名人名字缩写漏缺. 部分易混淆学名命名人 缩写中只有姓氏缩写, 而漏缺名字的缩写. 如《中国药 典》中收载熊胆基原动物黑熊(Selenaretos thibetanus Cuvier), 无法确定是同一时代的Frédéric Cuvier还是 Georges Cuvier. 对于命名人易混淆的分类群，缩写的 失误可能导致动物命名人引证的混乱，需保留命名人 必要的名字缩写, 如土鳖虫基原动物地鳖(Polyphaga 
表 2 《中国药典》中动物药材基原物种晚出名

\begin{tabular}{|c|c|c|c|c|}
\hline NO. & 品名 & 《中国药典》学名 & 完整引用 & 规范学名 \\
\hline 1 & 海马 & $\begin{array}{c}\text { 小海马(海蛆) (Hippocampus japonicus } \\
\text { Kaup) }\end{array}$ & $\begin{array}{l}\text { Hippocampus japonicus } \\
\text { Kaup, } 1856\end{array}$ & Hippocampus mohnikei Bleeker, 1853 \\
\hline 2 & 海螵蛸 & $\begin{array}{c}\text { 无针乌贼(Sepiella maindroni } \\
\text { de Rochebrune) }\end{array}$ & $\begin{array}{l}\text { Sepiella maindroni de } \\
\text { Rochebrune, } 1884\end{array}$ & Sepiella inermis (Van Hasselt, 1835) \\
\hline 3 & 蝉蜕 & $\begin{array}{c}\text { 黑蚱(Cryptotympana pustulata } \\
\text { Fabricius) }\end{array}$ & $\begin{array}{l}\text { Cryptotympana pustulata } \\
\text { (Fabricius, 1787) }\end{array}$ & Cryptotympana atrata (Fabricius, 1775) \\
\hline 4 & 夜明砂 & 东方蝙蝠(Vespertilio superans Thomas) & $\begin{array}{l}\text { Vespertilio superans } \\
\text { Thomas, } 1899\end{array}$ & Vespertilio sinensis (Peters, 1880) \\
\hline 5 & 虻虫 & 复带虻(Tabanus bivittatus Matsumura) & $\begin{array}{l}\text { Tabanus bivittatus } \\
\text { Matsumura, } 1916\end{array}$ & Atylotus miser (Szilady, 1915) \\
\hline
\end{tabular}

sinensis Walker, F., 1868), 方海(螃蟹)基原动物云南溪 蟹(Potamon denticulata H. Milne Edwards, 1853), 以及 五灵脂基原动物复齿鼠鼠(Trogopterus xanthipes (A. Milne-Edwards, 1867))等.

（4）同一基原物种命名人缩写不一致. 《中国药 典》中不同项下分类学家林奈的名称缩写不一致, 部 分缩写为L.而部分保持全称Linnaeus, 甚至对不同品 种项下的同一基原物种缩写也不一致. 如水牛角、水 牛角浓缩粉、牛心、牛角尖粉、牛乳项下水牛引证为 Bubalus bubalis Linnaeus, 而牛髓中水牛则引证为 $B u$ balus bubalis L., 蜂蜡和蜂蜜项下意大利蜂引证为Apis mellifera Linnaeus而蜂胶项下意大利蜂则引证为Apis mellifera L..

《中国药典》以实用性和稳定性为基本原则，国 家药典委员会在““中国药典2010版一部拉丁学名修 订'及 “生药拉丁名的词序变更' 等相关问题定专题审定 会纪要” 中指出, 中药基原植物定名人应采用缩写原 则, 但未确定基原动物的定名原则. 与植物命名人可 缩写的现状不同, 国际植物命名法规(International Code of Botanical Nomenclature, ICBN) 推荐植物学名 命名人的缩写形式以英国皇家植物园(Kew garden)发 布的标准索引为准, 其命名人缩写可形成共识, 确保 缩写对应唯一的命名人. 而国际动物命名法规第51条 规定：“命名者的姓名并不形成一个分类单元名称的 一部分, 引证时可以随意为之”, 并无一个强制或推荐 性的缩写标准, 故指明动物分类单元时通常引证其姓 氏全称, 建议《中国药典》对命名人的形式进行统一.

\section{5 拉丁学名缺失}

血余炭和乌鸡等两项缺失拉丁学名. 血余炭来源
于人科人(Homo sapiens Linnaeus, 1758)的头发, 乌鸡 来源于雉科家鸡(Gallus gallus (Linnaeus, 1758))的乌 骨鸡品种, 虽然都是常见物种, 从维护药典统一的角度 上, 也应引证相应的拉丁学名.

\section{6 亚属和第一修订人的引证问题}

除虫白蜡基原动物白蜡虫(Ericerus pela (Chavannes) Guerin)和五倍子基原动物五倍子蚜(Melaphis chinensis (Bell) Baker)引证了第一修订人, 方海(螃蟹) 基原动物溪蟹(Potamon (Potamon) denticulate) 和云南 溪蟹(Potamon (Potamon) yunanensis)及蟾酥、蟾皮、 干蟾基原动物(Bufo bufo gargarizans Cantor)引证了亚 属名, 其余物种均未引证亚属或第一修订人, 由于亚属 及第一修订人并非指明物种所必需的资料, 建议《中 国药典》取消引证这 4 种基原动物的亚属名或第一修 订人.

\section{7 驯养动物拉丁学名引证问题}

《动物学中适用名称目录》中指定了《中国药 典》涉及的6种驯养动物的拉丁学名并将其与野生种 进行了区分(表4). 根据国际动物命名法规第79条的要 求, 在涉及适用名称目录的物种时应以适用名称为准, 建议《中国药典》对已指定拉丁学名的物种引用均按 适用名称目录进行修订.

此外, 目前《中国药典》中所有动物药材基原物 种拉丁学名引证均缺少发表时间而不够规范. 与植物 药相比, 《中国药典》收载的动物药材基原物种的种 属分类已发生显著变化, 存在 30 余项学名的重新组 合、误用名的订正等. 故对《中国药典》涉及的 103 种动物药材基原物种拉丁学名进行修订, 并提供不改 
表 3 《中国药典》中组合改变引起的引证错误

\begin{tabular}{|c|c|c|c|}
\hline NO. & 药典拉丁名 & 完整引用 & 原始命名 \\
\hline 1 & 赛加羚羊(Saiga tatarica Linnaeus) & Saiga tatarica (Linnaeus, 1766) & Capra tatarica Linnaeus, 1766 \\
\hline 2 & 短刺猬(Hemichianus dauricus Sundevol) & Hemiechinus dauricus (Sundevall, 1842) & Erinaceus dauuricus Sundevall, 1842 \\
\hline 3 & 朝鲜黑金龟子(Holotrichia diomphalia Bates) & Holotrichia diomphalia (Bates, 1888) & Lachnosterna diomphalia Bates, 1888 \\
\hline 4 & 柳叶蚂蟥(Whitmania acranulata Whitman) & Whitmania acranulata (Whitman, 1886) & Leptostoma acranulata Whitman, 1886 \\
\hline 5 & 蚂蟥(Whitmania pigra Whitman) & Whitmania pigra (Whitman, 1884) & Leptostoma pigra Whitman, 1884 \\
\hline 6 & 黄黑小斑嵍(Mylabris cichorii Linnaeus) & Mylabris cichorii (Linnaeus, 1758) & Meloe cichorii Linnaeus, 1758 \\
\hline 7 & 南方大斑嵍(Mylabris phalerata Pallas) & Mylabris phalerata (Pallas, 1781) & Meloe phalerata Pallas, 1781 \\
\hline 8 & 地鳖(Eupolyphaga sinensis Walker) & Eupolyphaga sinensis (Walker F., 1868) & Polyphaga sinensis Walker, F., 1868 \\
\hline 9 & 屎壳螂(Catharsius molossus Linnaeus) & Catharsius molossus (Linnaeus, 1758) & Scarabaeus Molossus Linnaeus, 1758 \\
\hline 10 & 溪蟹(Potamon (Potamon) denticulata) & $\begin{array}{l}\text { Potamon denticulata } \mathrm{H} \text {. Milne-Edwards, } \\
1853\end{array}$ & $\begin{array}{c}\text { Thelphusa denticulata } \text { H. Milne-Edwards, } \\
1853\end{array}$ \\
\hline 11 & 水牛(Bubalus bubalis Linnaeus) & Bubalus bubalis (Linnaeus, 1758) & Bos Bubalis Linnaeus, 1758 \\
\hline 12 & 文蛤(Meretrix meretrix Linnaeus) & Meretrix meretrix (Linnaeus, 1758) & Venus meretrix Linnaeus, 1758 \\
\hline 13 & 青蛤(Cyclina sinensis Gmelin) & Cyclina sinensis (Gmelin, 1791) & Venus sinensis Gmelin, 1791 \\
\hline 14 & 蛤蚧 (Gekko gecko Linnaeus) & Gekko gecko (Linnaeus, 1758) & Lacerta gecko Linnaeus, 1758 \\
\hline 15 & 异腹胡蜂(Parapolybia varia Fabricius) & Parapolybia varia (Fabricius, 1787) & Vespa varia Fabricius, 1787 \\
\hline 16 & 黑虾(Cryptotympana pustulata Fabricius) & Cryptotympana pustulata (Fabricius, 1787) & Tettigonia pustulata Fabricius, 1787 \\
\hline 17 & 中华大蟾蜍(Bufo bufo gargarizans Cantor) & Bufo (Bufo) gargarizans Cantor, 1842 & Bufo gargarizans Cantor, 1842 \\
\hline 18 & $\begin{array}{c}\text { 复齿鼠鼠(Trogopterus xanthipes Milne-Ed- } \\
\text { wards) }\end{array}$ & Trogopterus xanthipes (A. Milne-Edwa & $\begin{array}{l}\text { Pteromys xanthipes A. } \\
\text { Milne-Edwards, } 1867\end{array}$ \\
\hline 19 & 短刺猬(Hemichianus dauricus Sundevol) & Hemiechinus dauricus (Sundevall, 1842) & Erinaceus dauuricus Sundevall, 1842 \\
\hline 20 & 黑熊(Selenaretos thibetanus Cuvier) & Selenarctos thibetanus (G. Cuvier, 1823) & Ursus thibetanus G. Cuvier, 1823 \\
\hline
\end{tabular}

表 4 《中国药典》与《动物学中适用名称目录》中驯养动物学名引证差异的比较

\begin{tabular}{|c|c|c|c|}
\hline NO. & 品名 & 药典拉丁名 & 适用名称目录拉丁名 \\
\hline 1 & 牛黄等 & 牛(Bos taurus domesticus Gmelin) & Bos taurus Linnaeus, 1758 \\
\hline 2 & 猪胆粉等 & 猪(Sus scrofa domestica Brisson) & Sus domesticus Erxleben, 1777 \\
\hline 3 & 僵蚕等 & 家虫(Bombyx mori Linnaeus) & Phalaena mori Linnaeus, 1758 \\
\hline 4 & 山羊角 & 山羊(Capra hircus L.) & Capra hircus Linnaeus, 1758 \\
\hline 5 & 羊骨等 & 绵羊(Ovis aries $\mathrm{L}$.) & Ovis aries Linnaeus, 1758 \\
\hline 6 & 狗骨等 & 狗(Canis familiaris L.) & Canis familiaris Linnaeus, 1758 \\
\hline
\end{tabular}

变《中国药典》种属组合引证，允许存在分类滞后的 “完整引证名”和根据公认的较新分类研究结果整理的 “推荐引证名”(表5).

\section{3 中文名引证的问题}

\section{1 同物异名}

为正确指明一种基原动物, 药典中拉丁学名与中
文名应统一. 如《中国药典》中牛黄、牛心、牛乳项 下引证Bos taurus domesticus Gmelin的中文名为“牛”, 而牛髓项下则引证为“黄牛”, 易引起混乱.

\section{2 易混淆名}

《中国药典》水蛭项下引证Whitmania pigra Whitman的中文名为“蚂蟥”, 引证Hirudo nipponica Whitman的中文名为“水蛭”, 而水蛭为蛭纲 (Hirudinea) 
表 5 中国药典中动物药材基原物种学名修订表 ${ }^{\text {a) }}$

\begin{tabular}{|c|c|c|c|c|}
\hline NO. & 品种 & 药典拉丁名 & 完整引证名 & 推荐引证名 \\
\hline 1 & 九香虫 ${ }^{*}$ & 九香虫(Aspongopus chinensis Dallas) & Aspongopus chinensis Dallas, 1851 & Coridius chinensis (Dallas, 1851) \\
\hline \multirow[t]{2}{*}{2} & 土鳖虫 ${ }^{*}$ & 地鳖(Eupolyphaga sinensis Walker) & Eupolyphaga sinensis (Walker F., 1868) & Eupolyphaga sinensis (Walker F., 1868) \\
\hline & & 冀地鳘(Steleophaga plancyi (Boleny)) & Steleophaga plancyi (Boleny, 1883) & Polyphaga plancyi Bolívar, 1883 \\
\hline \multirow[t]{3}{*}{3} & 瓦楞子* & 毛蚶(Arca subcrenata Lischke) & Arca subcrenata Lischke, 1869 & Anadara kagoshimensis (Tokunaga, 1906) \\
\hline & & 泥蚶(Arca granosa Linnaeus) & Arca granosa Linnaeus, 1758 & Tegillarca granosa (Linnaeus, 1758) \\
\hline & & 魁蚶(Arca inflata Reeve) & Arca inflata Reeve, 1844 & Anadara broughtonii (Schrenck, 1867) \\
\hline 4 & 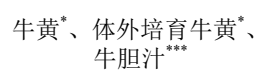 & 牛(Bos taurus domesticus Gmelin) & Bos taurus domesticus, Linnaeus, 1758 & Bos taurus Linnaeus, 1758 \\
\hline 5 & 乌梢蛇 ${ }^{*}$ & 乌梢蛇(Zaocys dhumnades (Cantor)) & Zaocys dhumnades (Cantor, 1842) & Ptyas dhumnades (Cantor, 1842) \\
\hline 6 & $\begin{array}{l}\text { 水牛角 }{ }^{*} \text { 、水牛角浓缩 } \\
\text { 粉 }^{*} \text { 、牛角尖粉 }\end{array}$ & 水牛(Bubalus bubalis Linnaeus) & Bubalus bubalis (Linnaeus, 1758) & Bubalus bubalis (Linnaeus, 1758) \\
\hline \multirow[t]{3}{*}{7} & 水蛭 ${ }^{*}$ & 蚂蟥(Whitmania pigra Whitman) & Whitmania pigra (Whitman, 1884) & Whitmania pigra (Whitman, 1884) \\
\hline & & 水蛭(Hirudo nipponica Whitman) & Hirudo nipponia Whitman, 1886 & Hirudo nipponia Whitman, 1886 \\
\hline & & $\begin{array}{c}\text { 柳叶蚂蟥(Whitmania acranulata Whit- } \\
\text { man) }\end{array}$ & Whitmania acranulata (Whitman, 1886) & Whitmania acranulata (Whitman, 1886) \\
\hline \multirow[t]{6}{*}{8} & 石决明 ${ }^{*}$ & 杂色鲍(Haliotis diversicolor Reeve) & Haliotis diversicolor Reeve, 1846 & Haliotis diversicolor Reeve, 1846 \\
\hline & & 皱纹盘鲍(Haliotis discus hannai Ino) & Haliotis discus hannai Ino, 1953 & Haliotis discus hannai Ino, 1953 \\
\hline & & 羊鲍(Haliotis ovina Gmelin) & Haliotis ovina Gmelin, 1791 & Haliotis ovina Gmelin, 1791 \\
\hline & & 澳洲鲍(Haliotis ruber (Leach)) & Haliotis rubra Leach, 1814 & Haliotis rubra Leach, 1814 \\
\hline & & 耳鲍(Haliotis asinina Linnaeus) & Haliotis asinina Linnaeus, 1758 & Haliotis asinina Linnaeus, 1758 \\
\hline & & 白鲍(Haliotis laevigata (Donovan)) & Haliotis laevigata Donovan, 1808 & Haliotis laevigata Donovan, 1808 \\
\hline \multirow[t]{4}{*}{9} & 地龙 ${ }^{*}$ & $\begin{array}{c}\text { 参环毛蚓(Pheretima aspergillum (E. } \\
\text { Perrier)) }\end{array}$ & Pheretima aspergillum (Perrier, 1872) & Amynthas aspergillum (Perrier, 1872) \\
\hline & & 通俗环毛蚓(Pheretima vulgaris Chen) & Pheretima vulgaris Chen, 1930 & Metaphire vulgaris vulgaris (Chen, 1930) \\
\hline & & $\begin{array}{c}\text { 威廉环毛蚓(Pheretima guillelmi (Mi- } \\
\text { chaelsen)) }\end{array}$ & Pheretima guillelmi (Michaelsen, 1895) & Metaphire guillelmi (Michaelsen, 1895) \\
\hline & & $\begin{array}{c}\text { 栉盲环毛蚓(Pheretima pectinifera } \mathrm{Mi} \text { - } \\
\text { chaelsen) }\end{array}$ & Pheretima pectinifera Michaelsen, 1931 & Amynthas pectiniferus (Michaelsen, 1931) \\
\hline 10 & 虫白蜡* & $\begin{array}{c}\text { 白蜡虫(Ericerus pela (Chavannes) Guer- } \\
\text { in) }\end{array}$ & $\begin{array}{l}\text { Ericerus pela (Chavannes, 1848) Guérin, } \\
1858\end{array}$ & Ericerus pela (Chavannes, 1848) \\
\hline 11 & 全蝎 ${ }^{*}$ & 东亚钳蝎(Buthus martensii Karsch) & Buthus martensii Karsch, 1879 & Mesobuthus martensii (Karsch, 1879) \\
\hline \multirow[t]{3}{*}{12} & 牡蚛 ${ }^{*}$ & 长牡蚛(Ostrea gigas Thunberg) & Ostrea gigas Thunberg, 1793 & Crassostrea gigas (Thunberg, 1793) \\
\hline & & $\begin{array}{c}\text { 大连湾牡蚛( Ostrea talienwhanensis } \\
\text { Crosse) })^{* * * *}\end{array}$ & Ostrea talienwhanensis Crosse, 1862 & Crassostrea talienwhanensis (Crosse, 1862) ${ }^{*}$ \\
\hline & & 近江牡蚛(Ostrea rivularis Gould) & Ostrea rivularis Gould, 1861 & Crassostrea rivularis (Gould, 1861) \\
\hline 13 & 龟甲* & 乌龟(Chinemys reevesii (Gray)) & Chinemys reevesii (Gray, 1831) & Mauremys reevesii (Gray, 1831) \\
\hline 14 & 阿胶 ${ }^{*}$ & 驴(Equus asinus L.) & Equus asinus Linnaeus, 1758 & Equus asinus Linnaeus, 1758 \\
\hline 15 & 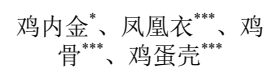 & 家鸡(Gallus gallus domesticus Brisson) & Gallus gallus domesticus Brisson, 1760 & Gallus gallus (Linnaeus, 1758) \\
\hline 16 & 金钱白花蛇 ${ }^{*}$ & 银环蛇(Bungarus multicinctus Blyth) & Bungarus multicinctus Blyth, 1861 & Bungarus multicinctus Blyth, 1861 \\
\hline \multirow[t]{3}{*}{17} & 珍珠 ${ }^{*}$ 珍珠母 ${ }^{*}$ & 马氏珍珠贝(Pteria martensii (Dunker)) & Pteria martensii (Dunker, 1872) & Pinctada martensii (Dunker, 1872) \\
\hline & & 三角帆蚌(Hyriopsis cumingii (Lea)) & Hyriopsis cumingii (Lea, 1852) & Hyriopsis cumingii (Lea, 1852) \\
\hline & & 褶纹冠蚌(Cristaria plicata (Leach)) & Cristaria plicata (Leach, 1815) & Cristaria plicata (Leach, 1815) \\
\hline 18 & 哈蟆油 ${ }^{*}$ & $\begin{array}{c}\text { 中国林蛙(Rana tem poraria chensinensis } \\
\text { David) }\end{array}$ & Rana chensinensis David, 1875 & Rana chensinensis David, 1875 \\
\hline 19 & 穿山甲* & 穿山甲(Manis pentadactyla Linnaeus) & Manis pentadactyla Linnaeus, 1758 & Manis pentadactyla Linnaeus, 1758 \\
\hline 20 & 海马* & $\begin{array}{c}\text { 线纹海马(Hippocampus kelloggi Jordan } \\
\text { et Snyder) }\end{array}$ & $\begin{array}{l}\text { Hippocampus kelloggi Jordan \& Snyder, } \\
1901\end{array}$ & Hippocampus kelloggi Jordan \& Snyder, 1901 \\
\hline
\end{tabular}




\begin{tabular}{|c|c|c|c|c|}
\hline NO. & 品种 & 药典拉丁名 & 完整引证名 & 推荐引证名 \\
\hline & & 刺海马(Hippocampus histrix Kaup) & Hippocampus histrix Kaup, 1856 & Hippocampus histrix Kaup, 1856 \\
\hline & & 大海马(Hippocampus kuda Bleeker) & Hippocampus kuda Bleeker, 1852 & Hippocampus kuda Bleeker, 1852 \\
\hline & & $\begin{array}{c}\text { 三斑海马(Hippocampus trimaculatus } \\
\text { Leach) }\end{array}$ & Hippocampus trimaculatus Leach, 1814 & Hippocampus trimaculatus Leach, 1814 \\
\hline & & $\begin{array}{c}\text { 小海马(海蛆) (Hippocampus japonicus } \\
\text { Kaup) }\end{array}$ & Hippocampus japonicus Kaup, 1856 & Hippocampus mohnikei Bleeker, 1853 \\
\hline \multirow[t]{3}{*}{21} & 海龙 ${ }^{*}$ & 海龙(Solenognathus hardwickii (Gray)) & Solegnathus hardwickii (Gray, 1830) & Solegnathus hardwickii (Gray, 1830) \\
\hline & & $\begin{array}{c}\text { 拟海龙(Syngnathoides biaculeatus } \\
\text { (Bloch)) }\end{array}$ & Syngnathoides biaculeatus (Bloch, 1785) & Syngnathoides biaculeatus (Bloch, 1785) \\
\hline & & 尖海龙(Syngnathus acus Linnaeus) & Syngnathus acus Linnaeus, 1758 & Syngnathus acus Linnaeus, 1758 \\
\hline \multirow[t]{2}{*}{22} & 海螵蛸* & $\begin{array}{c}\text { 无针乌贼(Sepiella maindroni de Rocheb- } \\
\text { rune) }\end{array}$ & Sepiella maindroni de Rochebrune, 1884 & Sepiella inermis (Van Hasselt, 1835) \\
\hline & & 金乌贼(Sepia esculenta Hoyle) & Sepia esculenta Hoyle, 1885 & Sepia esculenta Hoyle, 1885 \\
\hline \multirow[t]{3}{*}{23} & 桑螵蛸* & 大刀螂(Tenodera sinensis Saussure) & Tenodera sinensis Saussure, 1871 & Tenodera sinensis Saussure, 1871 \\
\hline & & 小刀螂(Statilia maculata (Thunberg)) & Statilia maculata (Thunberg, 1784) & Statilia maculata (Thunberg, 1784) \\
\hline & & $\begin{array}{c}\text { 巨斧螳螂(Hierodula patellifera (Ser- } \\
\text { ville)) }\end{array}$ & Hierodula patellifera (Serville, 1839) & Hierodula patellifera (Serville, 1839) \\
\hline \multirow[t]{3}{*}{24} & 蛇蜕* & 黑眉锦蛇(Elaphe taeniura Cope) & Elaphe taeniurus Cope, 1861 & Orthriophis taeniurus (Cope, 1861) \\
\hline & & 王锦蛇(Elaphe carinata (Guenther)) & Elaphe carinata (Günther, 1864) & Elaphe carinata (Günther, 1864) \\
\hline & & 乌梢蛇(Zaocys dhumnades (Cantor)) & Zaocys dhumnades (Cantor, 1842) & Ptyas dhumnades (Cantor, 1842) \\
\hline 25 & $\begin{array}{c}\text { 猪胆粉 }^{*} \text { 、猪骨 }{ }^{* * *} \text { 、猪胆 } \\
\text { 汁 }^{* * *} \text { 、猪脊髓 }{ }^{* * *} \text { 、猪脑 } \\
\text { 粉 }\end{array}$ & 猪(Sus scrofa domestica Brisson) & Sus scrofa domestica Brisson, 1760 & Sus domesticus Erxleben, 1777 \\
\hline \multirow[t]{2}{*}{26} & $\begin{array}{c}\text { 鹿角 }{ }^{*} 、 \text { 鹿茸* } 、 \text { 鹿心 } \\
\text { 粉 }^{* * *} 、 \text { 鹿血.** }^{* *}\end{array}$ & 马鹿(Cervus elaphus Linnaeus) & Cervus elaphus Linnaeus, 1758 & Cervus elaphus Linnaeus, 1758 \\
\hline & & 梅花鹿(Cervus nippon Temminck) & Cervus nippon Temminck, 1838 & Cervus nippon Temminck, 1838 \\
\hline 27 & 羚羊角 ${ }^{*}$ & 赛加羚羊(Saiga tatarica Linnaeus) & Saiga tatarica (Linnaeus, 1766) & Saiga tatarica (Linnaeus, 1766) \\
\hline \multirow[t]{2}{*}{28} & 斑蟼" & 南方大斑螫(Mylabris phalerata Pallas) & Mylabris phalerata (Pallas, 1781) & Mylabris phalerata (Pallas, 1781) \\
\hline & & 黄黑小斑沝(Mylabris cichorii Linnaeus) & Mylabris cichorii (Linnaeus, 1758) & Mylabris cichorii (Linnaeus, 1758) \\
\hline \multirow[t]{2}{*}{29} & 蛤壳* & 文蛤(Meretrix meretrix Linnaeus) & Meretrix meretrix (Linnaeus, 1758) & Meretrix meretrix (Linnaeus, 1758) \\
\hline & & 青蛤(Cyclina sinensis Gmelin) & Cyclina sinensis (Gmelin, 1791) & Cyclina sinensis (Gmelin, 1791) \\
\hline 30 & 蛤蚧* & 蛤蚧(Gekko gecko Linnaeus) & Gekko gecko (Linnaeus, 1758) & Gekko gecko (Linnaeus, 1758) \\
\hline 31 & 蜈蚣* & $\begin{array}{c}\text { 少棘巨蜈蚣(Scolopendra subspinipes } \\
\text { mutilans L. Koch) }\end{array}$ & $\begin{array}{c}\text { Scolopendra subspinipes mutilans L. } \\
\text { Koch, } 1878\end{array}$ & Scolopendra subspinipes mutilans L. Koch, 1878 \\
\hline \multirow[t]{3}{*}{32} & 蜂房* & 果马蜂(Polistes olivaceous (DeGeer)) & Polistes olivaceus (DeGeer, 1773) & Polistes olivaceus (DeGeer, 1773) \\
\hline & & $\begin{array}{c}\text { 日本长脚胡蜂(Polistes japonicus Saus- } \\
\text { sure) }\end{array}$ & Polistes japonicus Saussure, 1858 & Polistes japonicus Saussure, 1858 \\
\hline & & 异腹胡蜂(Parapolybia varia Fabricius) & Parapolybia varia (Fabricius, 1787) & Parapolybia varia (Fabricius, 1787) \\
\hline 33 & 蜂胶 ${ }^{*}$ & 意大利蜂(Apis mellifera $\mathrm{L}$. .) & Apis mellifera Linnaeus, 1758 & Apis mellifera Linnaeus, 1758 \\
\hline \multirow[t]{2}{*}{34} & 蜂蜡"、蜂蜜" & 中华蜜蜂(Apis cerana Fabricius) & Apis cerana Fabricius, 1793 & Apis cerana Fabricius, 1793 \\
\hline & & 意大利蜂(Apis mellifera Linnaeus) & Apis mellifera Linnaeus, 1758 & Apis mellifera Linnaeus, 1758 \\
\hline 35 & 蝉蜕 ${ }^{*}$ & 黑蚱(Cryptotympana pustulata Fabricius) & $\begin{array}{c}\text { Cryptotympana pustulata (Fabricius, } \\
1787 \text { ) }\end{array}$ & Cryptotympana atrata (Fabricius, 1775) \\
\hline 36 & 龩蛇 ${ }^{*}$ & 五步蛇(Agkistrodon acutus (Güenther)) & Agkistrodon acutus (Güenther, 1888) & Deinagkistrodon acutus (Günther, 1888) \\
\hline 37 & 僵蚕"、蚕沙 ${ }^{*}$ & 家蚕(Bombyx mori Linnaeus) & Bombyx mori Linnaeus, 1758 & Phalaena mori Linnaeus, 1758 \\
\hline \multirow[t]{2}{*}{38} & $\begin{array}{c}\text { 蟾酥 }{ }^{*} \text { 、蟾皮 } \\
\text { 干蟾 }^{* * * *} \text { 、 }\end{array}$ & $\begin{array}{c}\text { 中华大蟾蜍(Bufo bufo gargarizans Can- } \\
\text { tor) }\end{array}$ & Bufo (Bufo) gargarizans (Cantor, 1842) & Bufo gargarizans (Cantor, 1842) \\
\hline & & 黑眶蟾蜍(Bufo melanostictus Schneider) & Bufo melanostictus Schneider, 1799 & Duttaphrynus melanostictus (Schneider, 1799) \\
\hline 39 & 鳖甲* & 鳖(Trionyx sinensis Wiegmann) & Trionyx sinensis Wiegmann, 1834 & Pelodiscus sinensis (Wiegmann, 1834) \\
\hline
\end{tabular}




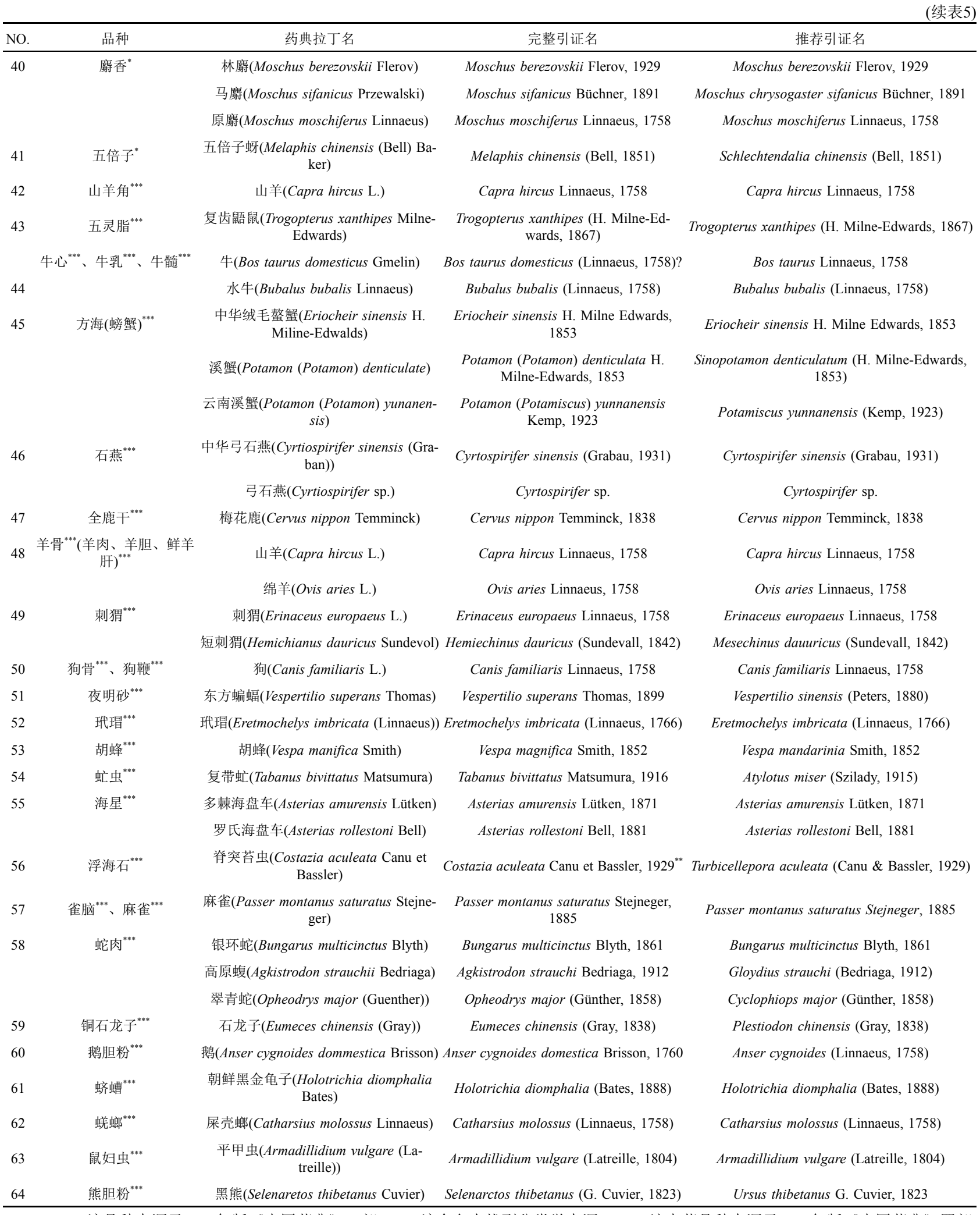

a) *: 该品种来源于 2015 年版《中国药典》一部; **: 该命名未找到分类学来源; ***: 该中药品种来源于 2015 年版《中国药典》四部; ****: 目前有分类学家认为大连湾牡蛎为长牡蚛的同物异名 


\section{表 6 《中国药典》动物药材基原物种中文名与分类学常用中文名对比}

\begin{tabular}{|c|c|c|}
\hline NO. & 药典中文名 & 分类学中文名 \\
\hline 1 & 参环毛蚓(Pheretima aspergillum (E. Perrier)) & 参状远盲蚂(Amynthas aspergillum (Perrier, 1872)) \\
\hline 2 & 通俗环毛蚓(Pheretima vulgaris Chen) & 通俗腔蚓(Metaphire vulgaris vulgaris (Chen, 1930)) \\
\hline 3 & 威廉环毛蚓(Pheretima guillelmi (Michaelsen)) & 威廉腔蚓(Metaphire guillelmi (Michaelsen, 1895)) \\
\hline 4 & 栉盲环毛蚂(Pheretima pectinifera Michaelsen) & 栉盲远盲蚂(Amynthas pectiniferus (Michaelsen, 1931)) \\
\hline 5 & 东亚钳蝎(Buthus martensii Karsch) & 马氏正钳蝎(Mesobuthus martensii (Karsch, 1879)) \\
\hline 6 & 马氏珍珠贝(Pteria martensii (Dunker)) & 马氏珠母贝(Pinctada martensii (Dunker, 1872)) \\
\hline 7 & 黑眉锦蛇(Elaphe taeniura Cope) & 黑眉晨蛇(Orthriophis taeniurus (Cope, 1861)) \\
\hline 8 & 五步蛇(Agkistrodon acutus (Güenther)) & 尖吻蝮(Deinagkistrodon acutus (Günther, 1888)) \\
\hline 9 & 鳖(Trionyx sinensis Wiegmann) & 中华鳖(Pelodiscus sinensis (Wiegmann, 1834)) \\
\hline 10 & 溪蟹(Potamon (Potamon) denticulata) & 锯齿华溪蟹(Sinopotamon denticulatum (H. Milne-Edwards, 1853)) \\
\hline 11 & 云南溪蟹(Potamon (Potamon) yunanensis) & 云南近溪蟹(Potamiscus yunnanensis (Kemp, 1923)) \\
\hline 12 & 短刺猬(Hemichianus dauricus Sundevol) & 达乌尔猬(Mesechinus dauuricus (Sundevall, 1842)) \\
\hline 13 & 复带虻(Tabanus bivittatus Matsumura) & 骚扰黄虻(Atylotus miser (Szilady, 1915)) \\
\hline 14 & 地鳖(Eupolyphaga sinensis Walker) & 中华真地鳖(Eupolyphaga sinensis (Walker, 1868)) \\
\hline 15 & 蚂蟥(Whitmania pigra Whitman) & 宽体金线蛭(Whitmania pigra (Whitman, 1884)) \\
\hline 16 & 水蛭(Hirudo nipponica Whitman) & 日本医蛭(Hirudo nipponia Whitman, 1886) \\
\hline 17 & 柳叶蚂蟥(Whitmania acranulata Whitman) & 尖细金线蛭(Whitmania acranulata (Whitman, 1886)) \\
\hline 18 & 赛加羚羊(Saiga tatarica Linnaeus) & 高鼻羚羊(Saiga tatarica (Linnaeus, 1766)) \\
\hline 19 & 南方大斑嵍(Mylabris phalerata Pallas) & 大斑芫菁(Mylabris phalerata (Pallas, 1781)) \\
\hline 20 & 黄黑小斑泴(Mylabris cichorii Linnaeus) & 眼斑芫菁(Mylabris cichorii (Linnaeus, 1758)) \\
\hline 21 & 蛤蚧(Gekko gecko Linnaeus) & 大壁虎(Gekko gecko (Linnaeus, 1758)) \\
\hline 22 & 中华蜜蜂(Apis cerana Fabricius) & 东方蜜蜂(Apis cerana Fabricius, 1793) \\
\hline 23 & 意大利蜂(Apis mellifera Linnaeus) & 西方蜜蜂(Apis mellifera Linnaeus, 1758) \\
\hline 24 & 中华线毛螯蟹(Eriocheir sinensis H. Miline-Edwalds) & 中华线螯蟹(Eriocheir sinensis H. Milne Edwards, 1853) \\
\hline 25 & 胡蜂(Vespa manifica Smith) & 金环胡蜂(Vespa mandarinia Smith, 1852) \\
\hline 26 & 朝鲜黑金龟子(Holotrichia diomphalia Bates) & 东北大黑鳃金龟(Holotrichia diomphalia (Bates, 1888)) \\
\hline 27 & 平甲虫(Armadillidium vulgare (Latreille)) & 普通卷甲虫(Armadillidium vulgare (Latreille, 1804)) \\
\hline 28 & 线纹海马(Hippocampus kelloggi Jordan et Snyder) & 克氏海马(Hippocampus kelloggi Jordan et Snyder, 1901) \\
\hline 29 & 大海马(Hippocampus kuda Bleeker) & 管海马(Hippocampus kuda Bleeker, 1852) \\
\hline 30 & 小海马(海蛆) (Hippocampus japonicus Kaup) & 日本海马(海蛆) (Hippocampus mohnikei Bleeker, 1853) \\
\hline 31 & 大刀螂(Tenodera sinensis Saussure) & 中华大刀螳(Tenodera sinensis Saussure, 1871) \\
\hline 32 & 小刀螂(Statilia maculata (Thunberg)) & 棕污斑螳(Statilia maculata (Thunberg, 1784)) \\
\hline 33 & 巨斧螳螂(Hierodula patellifera (Serville)) & 广斧螳(Hierodula patellifera (Serville, 1839)) \\
\hline 34 & 异腹胡蜂(Parapolybia varia Fabricius) & 变侧异腹胡蜂(Parapolybia varia (Fabricius, 1787)) \\
\hline 35 & 石龙子(Eumeces chinensis (Gray)) & 中国石龙子(Plestiodon chinensis (Gray, 1838)) \\
\hline 36 & 穿山甲(Manis pentadactyla Linnaeus) & 中华穿山甲(Manis pentadactyla Linnaeus, 1758) \\
\hline 37 & 五倍子蚜(Melaphis chinensis (Bell) Baker) & 角倍蚜(Schlechtendalia chinensis (Bell, 1851)) \\
\hline 38 & 中华线毛螯蟹(Eriocheir sinensis H. Miline-Edwalds) & 中华线螯蟹(Eriocheir sinensis H. Milne Edwards, 1853) \\
\hline 39 & 屎壳螂(Catharsius molossus Linnaeus) & 蛽螂(Catharsius molossus (Linnaeus, 1758)) \\
\hline 40 & 黑熊(Selenaretos thibetanus Cuvier) & 亚洲黑熊(Ursus thibetanus G. Cuvier, 1823) \\
\hline
\end{tabular}


动物的统称, 又称蚂蟥, 极易引起混乱, 建议将医蛭属 的Hirudo nipponica Whitman中文名修订为“日本医 蛭”, 将金线蛭属的W. pigra Whitman中文名修订为“宽 体金线蛭”, $W$. acranulata Whitman中文名修订为“尖细 金线蛭”. 海马基原动物Hippocampus kelloggi Jordan et Snyder的中文名“线纹海马”而国际上称“giant seahorse”，H. kuda Bleeker中文名“大海马”而国际上称 spotted seahorse, H. erectus Perry, 1810国际称“lined seahorse", 三者极易引起混淆，建议将 H. kelloggi中文 名修订为“克氏海马”, H. kuda中文名修订为“管海马”.

\section{3 组合改变导致的引证问题}

随着近年来分类学的发展, 动物物种出现亚种提 升到种，独立成为新的属或组合入其他属，属的拆分 或合并，其拉丁学名改变导致对应中文名变化. 《中 国药典》部分动物药材基原物种中文名与《中国动物 志》及专科属分类文献存在较大差异，如土鳖虫基原 物种地鳖组合入真地鳖属Eupolyphaga, 分类上称中华 真地鳖, 全蝎基原物种东亚钳蝎组合入正钳蝎属Mesobuthus, 分类上称马氏正钳蝎, 《中国药典》与分类学 文献同物异名详见表6.

\section{4 讨论}

名称是所指代物的外在体现，规范的名称有利于 准确指代事物的本质，先秦公孙龙在《指物论》中即 已提出：“天下无指者，生于物之各有名，不为指也”. 反映在中药上即准确的名称才能指代所用中药的实质 基原. 《中国药典》是我国药品监管领域的法定标准, 科学性、准确性是保障其权威性的前提. 在拉丁学名 引证方面，虽为保证应用上的延续性允许相对滞后， 但仍需确保引证规范和准确，以明确所规定中药的基 原. 因此, 建议在必要时, 国家药典委员会组织药典委 员和相关专家对《中国药典》基原物种信息进行全面 的审定.

相对于植物药基原物种有《中国植物志》、 《中 国高等植物》、Flora of China等3部主要分类学著作 可以参考, 目前我国并没有完备且权威, 涵盖药典中所
有动物药材基原物种的分类学著作. 《中国动物志》 尚未编制完成，其中部分早期编制的物种分类已超过 30年，部分分类结果已不符合近年来广为接受的分类 学观点, 而《中国药典》的基原物种分类则更滞后于 《中国动物志》的分类结果. 在国际学术交流, 如果 《中国药典》与其他国家的药典、草药典或膳食补充 剂法典的学名或分类体系不同，可能会造成一些误解 和不便. 虽然目前国际上已有几个物种名录信息的权 威数据库, 如Species 2000 的生物动态名录、 Global Names Architecture数据库、Global Biodiversity Information Facility数据库等, 但多基于生物多样性保护, 不 以提供名称信息为主要任务, 仅提供拉丁学名基本信 息，不能进一步提供命名上更为深入细致的信息. 因 此, 有必要建立一个针对《中国药典》基原物种的准 确学名数据库, 涵盖学名、原始命名、异名(synonym)、分类信息、作者引证及作者信息，以更好地指 明中药品种项下的真实物种基原.

另一方面, 随着动植物分类学的发展, 不同分类观 点不断被提出, 部分亚种提升成种, 分类学种的拆分, 或一些种被认为是另一些种的异名，对《中国药典》 基原物种引证提出了挑战. 如《中国药典》规定蜂蜜 等中药的基原物种为意大利蜂和中华蜜蜂(Apis cera$n a$ Fabricius), 而《中国动物志》则将中华蜜蜂处理为 东方蜜蜂中华亚种 $A$. cerana cerana Fabricius, 1793, 由 于东方蜜蜂还包括日本亚种 $A$. cerana japonica (Radoszkowski, 1877)和印度亚种 A. cerana indica (Fabricius, 1798)等13个不同亚种，《中国药典》规定的中华 蜜蜂所指称范围是整个东方蜜蜂物种 $A$. cerana Fabricius，1793亦或仅指中华亚种 $A$. cerana cerana Fabricius, 1793 存在疑义. 《中国药典》与《中国动物志》 等分类学著作中文名与拉丁学名指代不一的情况也同 样存在于意大利蜂(西方蜜蜂)、通俗环毛蚓(通俗腔蚓

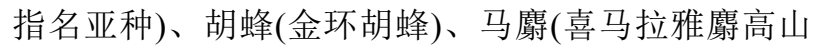
廭亚种)等. 为此，建议国家医药管理部门为《中国药 典》的所有基原物种建立实体标本库，建立并审定其 模式标本或模式标本群，从而为中药与其基原之间建 立起固定不变的联系，为中药基原及其拉丁学名正确 使用提供客观的参考标准. 


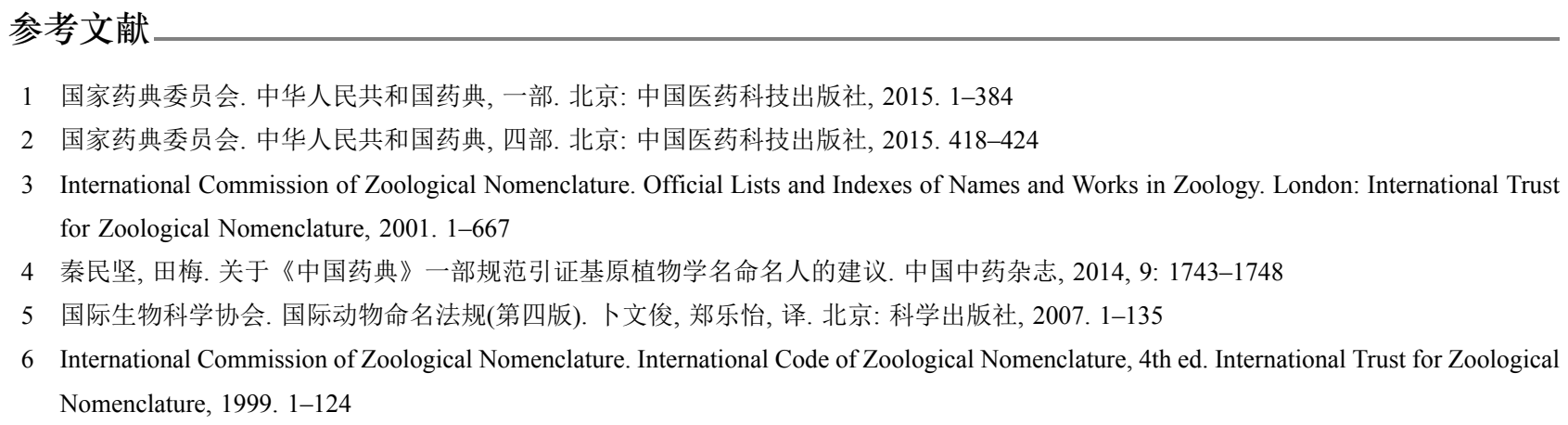

\title{
Standard citations of zoological scientific names and Chinese names in Chinese Pharmacopoeia
}

\begin{abstract}
JIANG Chao, HUANG LuQi, YUAN Yuan \& Li JunDe
State Key Laboratory of Dao-di Herbs Breeding Base, National Resource Center for Chinese Materia Medica, China Academy of Chinese Medical Sciences, Beijing 100700, China

Chinese Pharmacopoeia is the legal standard for drug administration in China. Scientific accuracy, normativity and authority are the fundamental attributes of Chinese Pharmacopoeia. However, historical non-standard zoological scientific name citations are gradually accumulating because of an absence of a comprehensive way of verifying these scientific names in every version of Chinese Pharmacopoeia. This paper compares the original animal scientific names in Chinese Pharmacopoeia with Fauna Sinica, Official Lists and Indexes of Names and Works in Zoology and several other international animal catalog databases. The statistics and analysis of this non-standard citation phenomena of original animal scientific names and Chinese names are recorded in the Chinese Pharmacopoeia (2015 edition) according to the principles of the fourth edition of the International Code of Zoological Nomenclature (ICZN) and, therefore propose critical revisions. The authors hope this paper will help the authors in accurately citing scientific names and aid the revision of non-standard name citations in Chinese Pharmacopoeia.
\end{abstract}

\section{Chinese Pharmacopoeia, medicinal animal, scientific name, Chinese name}

doi: $10.1360 / \mathrm{N} 052017-00254$ 